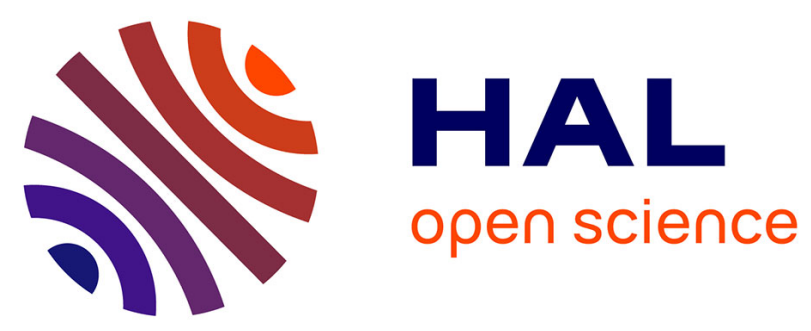

\title{
Closed-loop parametric identification for continuous-time linear systems via new algebraic techniques
}

\author{
Michel Fliess, Hebertt Sira-Ramirez
}

\section{- To cite this version:}

Michel Fliess, Hebertt Sira-Ramirez. Closed-loop parametric identification for continuous-time linear systems via new algebraic techniques. H. Garnier \& L. Wang. Identification of Continuoustime Models from Sampled Data, Springer, pp.362-391, 2008, Advances in Industrial Control, ISBN13:9781848001602. inria-00114958v3

\section{HAL Id: inria-00114958 \\ https://hal.inria.fr/inria-00114958v3}

Submitted on 17 Mar 2007

HAL is a multi-disciplinary open access archive for the deposit and dissemination of scientific research documents, whether they are published or not. The documents may come from teaching and research institutions in France or abroad, or from public or private research centers.
L'archive ouverte pluridisciplinaire $\mathbf{H A L}$, est destinée au dépôt et à la diffusion de documents scientifiques de niveau recherche, publiés ou non, émanant des établissements d'enseignement et de recherche français ou étrangers, des laboratoires publics ou privés. 


\title{
Closed-loop parametric identification for continuous-time linear systems via new algebraic techniques
}

\author{
Michel Fliess ${ }^{1}$ and Hebertt Sira-Ramirez ${ }^{2}$ \\ 1 Projet ALIEN, INRIA Futurs \\ \& Équipe MAX, LIX (CNRS, UMR 7161) \\ École polytechnique, 91128 Palaiseau, France \\ Michel.Fliess@polytechnique.edu \\ 2 Cinvestav-IPN \\ Depto. de Ing. Eléctrica, \\ Av. IPN, No. 2508, Col. San Pedro Zacatenco AP 14740 \\ 07300 México D.F., México. \\ hsira@cinvestav.mx
}

Summary. Closed-loop parametric identification of continuous-time linear systems is achieved via new algebraic techniques, which are employing non-commutative ring theory, module theory, and operational calculus. The efficiency and robustness with respect to noises are demonstrated by three illustrative examples and their computer simulations. In two of them we compare our results with those given by standard adaptive methods.

\section{Introduction}

A few years ago the two authors launched [11] a new approach to parametric identification of linear continuous-time systems. Its main features may be summarized as follows:

- Closed-loop identification is permitted thanks to the real-time identification scheme.

- The robustness with respect to noisy data is obtained without knowing the statistical properties of the corrupting noises.

These notes are devoted to a new exposition of those methods and to their illustration via three examples and their computer simulations.

Our mathematical techniques are quite different from those in the huge literature on this subject. We are mainly employing algebraic tools:

1. the module-theoretic approach to linear systems, 
2. elementary non-commutative ring theory,

3. operational calculus.

Our chapter is organized as follows. Section 2 gives a short summary of the module-theoretic setting for continuous-time linear systems ${ }^{3}$. Section 3 defines linear identifiability, which is sufficient for most practical cases, by introducing the notion of algebraic derivatives and the corresponding noncommutative ring theory. Section 4 is discussing two kinds of perturbations:

1. The structured perturbations, which satisfy time-varying linear differential equations, are annihilated by suitable linear differential operators.

2. The unstructured perturbations are considered as highly fluctuating, or oscillating, phenomena. They are attenuated by suitable low-pass filters, like iterated time integrals.

We thus arrive at estimators which are robust with respect to a large class of noises. Our numerical simulations in Sections 5 and 6 deal respectively with the open loop dragging of an unknown mass and the feedback control of a first order system. They are compared to standard adaptive methods, which seem to be less efficient. We note also in Section 5 that the rather complex notion of persistently exciting signal becomes quite pointless in our setting. For the double bridge buck converter of Section 7, which is more realistic (see [30]) than the previous examples, we are able to achieve a rather successful closed-loop parametric identification. A short conclusion relates our work to others.

Let us add that we tried to write the examples in such a way that they might be grasped without the necessity of reading the sections on the algebraic background. Our standpoint on parametric identification should therefore be accessible to most engineers.

\section{A module-theoretic approach to linear systems: a short summary}

\subsection{Some basic facts about modules over principal ideal rings}

Let $k$ be a given field ${ }^{4}$. Write $k[s]$ the ring of polynomials $\sum_{\text {finite }} a_{\nu} s^{\nu}, a_{\nu} \in k$, in the indeterminate $s$. It is well known that $k[s]$ is a principal ideal ring, i.e., any ideal of $k[s]$ may be generated by a single element. A $k[s]$-module $M$ is said to be finitely generated, or of finite type, if, and only if, $M=\operatorname{span}_{k[s]}(S)$,

\footnotetext{
${ }^{3}$ This module-theoretic presentation of linear systems started in [4]. See [2] for an excellent introduction and related references. This standpoint provides a most useful way for synthesizing model-based predictive control, which employs concepts stemming from flatness-based control [9, 28].

${ }^{4}$ See, e.g., [17] for a classic and well written introduction to commutative algebra.
} 
where $S$ is a finite set. Module $M$ is said to be $f r e e^{5}$ if, and only if, there exists $S$ whose elements are $k[s]$-linearly independent; $S$ is then called a basis. The cardinalities, i.e., the numbers of elements, of two bases are equal. Any submodule of a finitely generated (resp. free) $k[s]$-module is again finitely generated (resp. free). Any quotient module of a finitely generated $k[s]$-module is again finitely generated.

An element $x \in M$ is said to be torsion if, and only if, there exists $\varpi \in k[s]$, $\varpi \neq 0$, such that $\varpi x=0$. The set of all torsion elements of $M$ is a submodule $M^{\text {tor }},\{0\} \subseteq M^{\text {tor }} \subseteq M$, which is called the torsion submodule. If $M^{\text {tor }}=M$, $M$ is said to be torsion. If $M^{\text {tor }}=\{0\}, M$ is said to torsion-free. Any free module is of course torsion-free. As well known, the converse holds true for finitely generated torsion-free $k[s]$-module $M$. The quotient module $M / M^{\text {tor }}$ is free. The next property of a finitely generated $k[s]$-module $\mathrm{M}$ is crucial:

$$
M=M^{\text {tor }} \oplus F
$$

where the free module $F=M / M^{\text {tor }}$ is defined up to isomorphism.

A module, which is generated by a single element $g$, is finite-dimensional, when viewed as a $k$-vector space, if, and only if, $g$ is torsion. The extension to a finitely generated module $M$ is immediate: $M$ is torsion if, and only if, the dimension $\operatorname{dim}_{k}(M)$ of $M$, viewed as a $k$-vector space, is finite.

Example 1. Consider the set of $k[s]$-linear equations

$$
\sum_{\kappa=1}^{\mu} a_{\iota \kappa} \xi_{\kappa}=0, \quad a_{\iota \kappa} \in k[s], \iota=1, \ldots, \nu
$$

in the unknowns $\xi_{1}, \ldots, \xi_{\mu}$. Let $F$ be the free $k[s]$-module with basis $\left(f_{1}, \ldots\right.$, $\left.f_{\mu}\right)$. Let $E$ be the submodule generated by $e_{\iota}=\sum_{\kappa=1}^{\mu} a_{\iota \kappa} f_{\kappa}, \iota=1, \ldots, \nu$. Then, the module corresponding to equations (2) is $M=F / E$. Equations (2) may be written in the following matrix form

$$
P_{M}\left(\begin{array}{c}
\xi_{1} \\
\vdots \\
\xi_{\mu}
\end{array}\right)=0
$$

$P_{M} \in k[s]^{\nu \times \mu}$ is a presentation matrix of $\Lambda$.

\subsection{Formal Laplace transform}

Let $k(s)$ be the quotient field of $k[s]$, i.e., the field of rational functions over $k$ in the indeterminate $s$. Let $M$ be a finitely generated $k[s]$-module. The elements of the tensor product $\hat{M}=k(s) \otimes_{k[s]} M$ are finite sums of products $q^{-1} x$, $x \in M, q \in k[s], q \neq 0$. It is a $k(s)$-vector space, called the transfer vector

\footnotetext{
${ }^{5}$ By convention the trivial module $\{0\}$, generated by the empty set $\emptyset$, is free.
} 
space of $M$. The $k[s]$-linear mapping $M \rightarrow \hat{M}, m \mapsto \hat{m}=1 \otimes m$, is the formal Laplace transform ${ }^{6}$. Its kernel is the torsion submodule $M^{\text {tor }}$. The formal Laplace transform is thus injective if, and only if, $M$ is free. By definition, the rank of $M$, which is written $\operatorname{rk}(M)$, is $\operatorname{rk}(M)=\operatorname{dim}_{k(s)}(\hat{M})$. It is clear that $M$ is torsion if, and only if, $\operatorname{rk}(M)=0$. Take two modules $M_{1}, M_{2}, M_{1} \subseteq M_{2}$. Then, $\operatorname{rk}\left(M_{2} / M_{1}\right)=\operatorname{rk}\left(M_{2}\right)-\operatorname{rk}\left(M_{1}\right)$. Thus, $\operatorname{rk}\left(M_{1}\right)=\operatorname{rk}\left(M_{2}\right)$ if, and only if, the quotient module $M_{2} / M_{1}$ is torsion. For any set $\boldsymbol{x}=\left(x_{1}, \ldots, x_{\alpha}\right) \subset M$, the following equality is obvious:

$$
\operatorname{rk}\left(\operatorname{span}_{k[s]}(\boldsymbol{x})\right)=\operatorname{dim}\left(\operatorname{span}_{k(s)}(\hat{\boldsymbol{x}})\right)
$$

The next property is stating a useful matrix characterisation of torsion modules:

Proposition 1. The module corresponding to equation (3) is torsion if, and only if, $r k\left(P_{M}\right)=\mu$. If (3) is square, i.e., $\mu=\nu$, this condition is equivalent to $\operatorname{det}\left(P_{M}\right) \neq 0$.

Proof. The formal Laplace transform yields:

$$
P_{M}\left(\begin{array}{c}
\hat{\xi}_{1} \\
\vdots \\
\hat{\xi}_{\mu}
\end{array}\right)=0
$$

The module is torsion if, and only if, $\hat{\xi}_{1}=\ldots \hat{\xi}_{n}=0$. This latter condition is equivalent to $\mathrm{rk}\left(P_{M}\right)=\mu$.

Example 2. Let $T$ be a finitely generated torsion $k[s]$-module. Then, $\operatorname{dim}_{k}(T)=$ $n<\infty$. Pick up a basis $\boldsymbol{b}=\left(b_{1}, \ldots, b_{n}\right)$ of $T$ viewed as a $k$-vector space. To the $k$-linear mapping $s: T \rightarrow T, \tau \mapsto s \tau$, corresponds the matrix $A \in k^{n \times n}$ with respect to $\boldsymbol{b}$. This is equivalent saying that $T$ is defined by the following matrix equation:

$$
s\left(\begin{array}{c}
b_{1} \\
\vdots \\
b_{n}
\end{array}\right)=A\left(\begin{array}{c}
b_{1} \\
\vdots \\
b_{n}
\end{array}\right)
$$

It is clear that $\operatorname{det}(s-A) \neq 0$.

\subsection{Basic system-theoretic definitions}

A $k$-linear system is a finitely generated free $k[s]$-module $\Lambda$ where we have distinguished a finite subset of perturbation, or disturbance, variables $\underline{\boldsymbol{\pi}}=$ $\left(\underline{\pi}_{1}, \ldots, \underline{\pi}_{r}\right)$.

\footnotetext{
${ }^{6}$ See [5] for more details.
} 
Remark 1. Set $k=\mathbb{C}$. Consider the operational equation $a \chi=0, a \in \mathbb{C}[s], a \neq$ 0 , in the unknown $\chi$. Its unique solution is $\chi=0$. It means that any torsion element would be trivial. Note moreover that the linear differential equation $\dot{x}=0$, which corresponds to a torsion $\mathbb{C}\left[\frac{d}{d t}\right]$-module, yields the operational equation $s \hat{x}-x(0)=0$, which corresponds to a free $\mathbb{C}[s]$-module with basis $\{\hat{x}\}$. See $[10]$ for a thorough discussion.

The nominal, or unperturbed, system $\Lambda^{\text {nom }}$ is defined by the quotient module

$$
\Lambda^{\mathrm{nom}}=\Lambda / \operatorname{span}_{k[s]}(\underline{\boldsymbol{\pi}})
$$

The canonical image of any $\lambda \in \Lambda$ is written $\lambda^{\text {nom }} \in \Lambda^{\text {nom }}$. We might sometimes call $\Lambda$ a perturbed system. Note moreover that $\Lambda^{\text {nom }}$ is not necessarily free.

Example 3. The module corresponding to $s x=\underline{\pi}$, where $\underline{\pi} \in \operatorname{span}_{k[s]}(\underline{\boldsymbol{\pi}})$, $\underline{\pi} \neq 0$, is free ${ }^{7}$. The module corresponding to the nominal system $s x^{\text {nom }}=0$ is torsion.

A $k$-linear dynamics is a $k$-linear system $\Lambda$, which is equipped with a finite set $\boldsymbol{u}=\left(u_{1}, \ldots, u_{m}\right)$ of control variables, such that

- The control variables do not interact with the perturbation variables:

$$
\operatorname{span}_{k[s]}(\boldsymbol{u}) \cap \operatorname{span}_{k[s]}(\underline{\boldsymbol{\pi}})=\{0\}
$$

- The quotient module $\Lambda^{\mathrm{nom}} / \operatorname{span}_{k[s]}\left(\boldsymbol{u}^{\mathrm{nom}}\right)$ is torsion.

If $\boldsymbol{u}=\emptyset$, this last condition implies that $\Lambda^{\text {nom }}$ is torsion. The control variables are said to be independent if, and only if, $u_{1}, \ldots, u_{m}$ are $k[s]$-linearly independent.

The set of output variables is a finite subset $\boldsymbol{y}=\left(y_{1}, \ldots, y_{p}\right) \subset \Lambda$. A dynamics $\Lambda$ with output variables is called a $k$-linear input-output system. System is said to be mono-variable if, and only if, $m=p=1$. If not, it is said to be multi-variable.

\subsection{Transfer matrices}

Consider the nominal dynamics $\Lambda^{\text {nom }}$ with control variables

$$
\boldsymbol{u}^{\mathrm{nom}}=\left(u_{1}^{\mathrm{nom}}, \ldots, u_{m}^{\mathrm{nom}}\right)
$$

The transfer $k(s)$-vector space (see section 2.2) $\hat{\Lambda}^{\text {nom }}$ is spanned by $\hat{\boldsymbol{u}}^{\text {nom }}$, which is a basis if the control variables are independent. It yields with nominal output variables $\boldsymbol{y}^{\text {nom }}=\left(y_{1}^{\text {nom }}, \ldots, y_{p}^{\text {nom }}\right)$ :

\footnotetext{
${ }^{7}$ The initial condition $x(0)$ should be considered as a perturbation (see [10]).
} 


$$
\left(\begin{array}{c}
\hat{y}_{1}^{\mathrm{nom}} \\
\vdots \\
\hat{y}_{p}^{\text {nom }}
\end{array}\right)=T\left(\begin{array}{c}
\hat{u}_{1}^{\mathrm{nom}} \\
\vdots \\
\hat{u}_{m}^{\mathrm{nom}}
\end{array}\right)
$$

where $T \in k(s)^{p \times m}$ is the (nominal) transfer matrix, which is uniquely defined if, and only if, the control variables are independent. If $m=p=1, T$ is called a (nominal) transfer function. Matrix $T$ is said to be proper (resp. strictly proper) if, and only if, its entries are proper (resp. strictly proper) rational functions.

\section{Identifiability}

\subsection{Uncertain parameters}

Let the field $k$ be a finite algebraic extension ${ }^{8}$ of $k_{0}(\boldsymbol{\Theta})$, where

- $k_{0}$ is a given ground field,

- $\boldsymbol{\Theta}=\left(\theta_{1}, \ldots, \theta_{\tau}\right)$ is a finite set of uncertain, or unknown, parameters.

\subsection{The algebraic derivative and a new module structure}

Call ${ }^{9}$ with $[21,22,32]$ the derivation $\frac{d}{d s}$ with respect to $s$ the algebraic derivative $^{10}$. Introduce a new commutative field $K$ of constants, i.e., $\forall \xi \in K, \frac{d \xi}{d s}=0$. The ring $K\left[s, \frac{d}{d s}\right]$ of linear differential operators $\sum_{\text {finite }} a_{\nu} \frac{d^{\nu}}{d s^{\nu}}, a_{\nu} \in K[s]$, with polynomial coefficients, is called the Weyl algebra (see, e.g., [20]). It is noncommutative, as shown by the commutator $\left[\frac{d}{d s}, s\right]$ :

$$
\left[\frac{d}{d s}, s\right]=\frac{d}{d s} s-s \frac{d}{d s}=1
$$

Introduce the over-ring $K(s)\left[\frac{d}{d s}\right]$ of linear differential operators $\sum_{\text {finite }} b_{\nu} \frac{d^{\nu}}{d s^{\nu}}$, $b_{\nu} \in K(s)$, with rational coefficients. It is a non-commutative left and right principal ideal ring (see, e.g., [20]), i.e., any left (resp. right) ideal of $K(s)\left[\frac{d}{d s}\right]$ may be generated by a single element. Take again system $\Lambda$, i.e., a finitely generated free $k[s]$-module. Elements of the tensor product $\Lambda_{k(s)\left[\frac{d}{d s}\right]}=k_{0}(s)\left[\frac{d}{d s}\right] \otimes_{k[s]} \Lambda$ are (see, e.g., [20]) finite sums of products $r \lambda$, $r \in k_{0}(s)\left[\frac{d}{d s}\right], \lambda \in \Lambda$. It means that $\Lambda_{k(s)\left[\frac{d}{d s}\right]}$ may be endowed with a structure of left $k(s)\left[\frac{d}{d s}\right]$-module.

\footnotetext{
${ }^{8}$ A field extension $L / K$ is given by two fields $K$ and $L$ such that $K \subseteq L$ (see, e.g., [17]). It is a finite algebraic extension if, and only if, the dimension of $L$ viewed as a vector space over $K$ is finite. Then any element of $L$ is algebraic over $K$, i.e., satisfies a polynomial equation with coefficients in $K$.

${ }^{9}$ See $[8,10,11]$ for more details.

${ }^{10}$ Remember (see, e.g., $\left.[3,21,22,25,32]\right)$ that $\frac{d}{d s}$ corresponds in the time domain to the multiplication by $-t$.
} 


\subsection{Linear identifiability}

The uncertain parameters $\boldsymbol{\Theta}=\left(\theta_{1}, \ldots, \theta_{\tau}\right)$ are said to be linearly identifiable if, and only if,

$$
P\left(\begin{array}{c}
\theta_{1} \\
\vdots \\
\theta_{\tau}
\end{array}\right)=Q+R
$$

where

- the entries of the matrices $P$ and $Q$, of respective sizes $\tau \times \tau$ and $\tau \times 1$, belong to $\operatorname{span}_{k_{0}(s)\left[\frac{d}{d s}\right]}(\boldsymbol{u}, \boldsymbol{y})$,

- $\operatorname{det}(P) \neq 0$,

- $R$ is a $\tau \times 1$ matrix with entries in $\operatorname{span}_{k(s)\left[\frac{d}{d s}\right]}(\underline{\boldsymbol{\pi}})$.

The uncertain parameters $\Theta$ are said to be projectively linearly identifiable if, and only if,

- it is known that $\theta_{\iota} \neq 0$ for some $\iota, 1 \leq \iota \leq \tau$,

- the quantities $\left\{\frac{\theta_{1}}{\theta_{\iota}}, \ldots, \frac{\theta_{\iota-1}}{\theta_{\iota}}, \frac{\theta_{\iota+1}}{\theta_{\iota}}, \ldots, \frac{\theta_{\tau}}{\theta_{\iota}}\right\}$ are linearly identifiable ${ }^{11}$.

The uncertain parameters $\Theta$ are said to be weakly linearly identifiable if, and only if, there exists a set $\boldsymbol{\Theta}^{\prime}=\left\{\theta_{1}^{\prime}, \ldots, \theta_{\tau}^{\prime}\right\}$ of linearly identifiable quantities such that the elements of $\Theta$ are algebraic over $k_{0}\left(\boldsymbol{\Theta}^{\prime}\right)$.

\subsection{An elementary example}

Set $k_{0}=\mathbf{Q}$ and $\boldsymbol{\Theta}=\left\{a_{1}, \ldots, a_{n}, b_{0}, \ldots, b_{m}\right\}$. Consider the SISO system

$$
\left(\frac{d^{n}}{d t^{n}}+a_{1} \frac{d^{n-1}}{d t^{n-1}}+\cdots+a_{n}\right) y(t)=\left(b_{0} \frac{d^{m}}{d t^{m}}+\cdots+b_{m}\right) u(t)
$$

which reads with operational notations

$$
\left(s^{n}+a_{1} s^{n-1}+\cdots+a_{n}\right) Y=\left(b_{0} s^{m}+\cdots+b_{m}\right) U+\mathcal{I}(s)
$$

where $\mathcal{I}(s)$ is a polynomial over $k$ in the indeterminate $s$, of degree $\max (m, n)-$ 1 , the coefficients of which depend on the initial conditions. By applying $\frac{d^{\max (m, n)}}{d s^{\max (m, n)}}$ to both sides of Equation (10), we get rid of those conditions. The linear identifiability follows at once from the linear equations

$$
\frac{d^{\alpha}}{d s^{\alpha}}\left(s^{n}+a_{1} s^{n-1}+\cdots+a_{n}\right) Y=\frac{d^{\alpha}}{d s^{\alpha}}\left(b_{0} s^{m}+\cdots+b_{m}\right) U
$$

for $\max (m, n) \leq \alpha \leq \max (m, n)+m+n$.

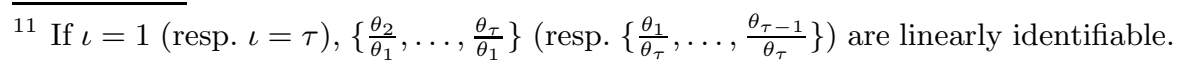


Remark 2. See [31] for most interesting calculations via moments which bear some similarity with the above ones.

Remark 3. Replace Equation (9) by

$$
\left(a_{0} \frac{d^{n}}{d t^{n}}+a_{1} \frac{d^{n-1}}{d t^{n-1}}+\cdots+a_{n}\right) y(t)=\left(b_{0} \frac{d^{m}}{d t^{m}}+\cdots+b_{m}\right) u(t)
$$

where we have introduced the coefficient $a_{0}$. If we assume for instance that $a_{0} \neq 0$, the set $\left\{a_{0}, a_{1}, \ldots, a_{n}, b_{0}, \ldots, b_{m}\right\}$ is obviously not linearly identifiable but projectively linearly identifiable.

\section{Perturbations}

\subsection{Structured perturbations}

For dealing with specific perturbations, introduce the left $k(s)\left[\frac{d}{d s}\right]$-module

$$
\mathbf{L}=\Lambda_{k(s)\left[\frac{d}{d s}\right]} / M
$$

where $M$ is a submodule of $\operatorname{span}_{k(s)\left[\frac{d}{d s}\right]}(\underline{\boldsymbol{\pi}})$. Call again perturbation, or disturbance, variables the canonical image $\boldsymbol{\pi}=\left(\pi_{1}, \ldots, \pi_{q}\right) \subset \mathbf{L}$ of $\underline{\boldsymbol{\pi}}$. A subset $S \subseteq \pi$ is said to be structured if, and only if, the module $\operatorname{span}_{k(s)\left[\frac{d}{d s}\right]}(S)$ is torsion. It means in other words that for any $\sigma \in \operatorname{span}_{k(s)\left[\frac{d}{d s}\right]}(S)$, there exists $\varpi \in k(s)\left[\frac{d}{d s}\right], \varpi \neq 0$, such that $\varpi \sigma=0$. We say that the linear differential operator $\varpi$ is annihilating the structured perturbation $\sigma$. The differential operator $\varpi$ is also called an annihilator of $\sigma$.

Example 4. Set $k=\mathbb{C}$. The perturbation $\kappa \frac{\mathrm{e}^{-L s}}{s^{n}}, L \geq 0, \kappa \in \mathbb{C}, n \geq 0$, which is annihilated by $\left(\frac{d}{d s}+L\right) s^{n}=s^{n}\left(\frac{d}{d s}+L\right)+n s^{n-1}$, is structured. Note that the annihilating differential operator contains $L$, but not $\kappa$.

Example 5. The perturbation $\frac{a}{b}, a, b \in k[s], b \neq 0$, which is annihilated by $\frac{d^{\nu}}{d s^{\nu}} b$, for $\nu$ large enough, is structured. In particular $\frac{\alpha s+\beta}{s^{2}+\omega^{2}}, \alpha, \beta, \omega \in k$, is annihilated by $\frac{d^{2}}{d s^{2}}\left(s^{2}+\omega^{2}\right)=2+2 s \frac{d}{d s}+\left(s^{2}+\omega^{2}\right) \frac{d^{2}}{d s^{2}}$, which contains the "frequency" $\omega$, but not $\alpha$ and $\beta$.

The set of annihilators of any $\sigma \in \operatorname{span}_{k(s)\left[\frac{d}{d s}\right]}(S)$ is a left ideal of $k(s)\left[\frac{d}{d s}\right]$. Any generator $\varpi_{0}$ of this principal ideal is said to be a minimal annihilator. Take two minimal annihilators $\varpi_{0}$ and $\varpi_{1}$. Then, $\varpi_{1}=\varrho \varpi_{0}$, where $\varrho \in k(s)$, $\varrho \neq 0$. 


\subsection{Unstructured perturbations}

Perturbations which are not structured are said to be unstructured. Such noises are viewed as highly fluctuating, or oscillatory, signals, which may be attenuated by low-pass filters, like iterated time integrals.

Remark 4. See [6] for a precise mathematical foundation, which is based on nonstandard analysis. A highly fluctuating function of zero mean is then defined by saying that its integral over a finite time interval is infinitesimal, i.e., "very small". Let us emphasize once more that this approach, which has been confirmed by numerous computer simulations and several laboratory experiments, is independent of any probabilistic setting. No knowledge of the statistical properties of the noises is required.

\subsection{Linear identifier}

Equation (8) may be rewritten as

$$
P\left(\begin{array}{c}
\theta_{1} \\
\vdots \\
\theta_{\tau}
\end{array}\right)=Q+R^{\text {struc }}+R^{\text {unstruc }}
$$

where the components of the column matrix $R^{\text {struc }}$ (resp. $\left.R^{\text {unstruc }}\right)$ are structured (resp. unstructured) perturbations. The set $\operatorname{ann}\left(R^{\text {struc }}\right)$ of differential polynomials $\omega \in k(s)\left[\frac{d}{d s}\right]$ annihilating $R^{\text {struc }}$, i.e., such that $\omega R^{\text {struc }}=0$, is a left ideal. Two generators $\Delta_{1}, \Delta_{2}$ of this ideal are related by $\Delta_{2}=\rho \Delta_{1}$, $\rho \in k(s), \rho \neq 0$. Pick up a generator $\Delta$ :

$$
\Delta P\left(\begin{array}{c}
\theta_{1} \\
\vdots \\
\theta_{\tau}
\end{array}\right)=\Delta Q+\Delta R^{\text {unstruc }}
$$

If $\operatorname{det}(\Delta P) \neq 0$, Equation (11), where the structured perturbations have been eliminated, is called a linear identifier of the unknown parameters.

\subsection{Robustness}

By multiplying both sides of Equation (11) by a suitable strictly proper transfer function in $k(s)$, we may ensure that any entry of the matrices is a $k$-linear combination of terms of the form $r \frac{d^{\alpha}}{d s^{\alpha}}(a)$, where

- $r \in k(s)$ is strictly proper,

- $\alpha=0,1,2, \ldots$,

- $a$ is either a control, an output, or an unstructured perturbation variable.

Denoising, i.e., the attenuation of unstructured perturbations, is achieved by choosing appropriate low-pass filters, like iterated time integrals, which give rise to what we may call invariant filtering. 


\section{First example: Dragging an unknown mass in open loop}

\subsection{Description and first results}

Consider the problem of dragging an unknown mass along a frictionless horizontal straight line. The model is given by

$$
m \ddot{x}(t)=u(t)
$$

where

- $x(t)$ is the mass displacement, perfectly measured from some reference point, or origin, labeled by 0 ,

- $u(t)$ is the applied force.

To make the problem simple, let us assume that $u(t)$ is a known, non-zero, open loop control force at our disposal. The entire purpose of applying such a force to the mass is to gather some input-output information so that we can identify the unknown mass parameter $m$. The mass is initially, at time $t=0$, placed at a distance $x_{0}$ of the origin and moves with unknown velocity $\dot{x}_{0}$.

Operational calculus yields

$$
m\left[s^{2} X-s x_{0}-\dot{x}_{0}\right]=U
$$

The dependence of this expression upon the initial conditions is eliminated by differentiating both sides twice with respect to $s$ :

$$
m\left[2 X+4 s \frac{d X}{d s}+s^{2} \frac{d^{2} X}{d s^{2}}\right]=\frac{d^{2} U}{d s^{2}}
$$

Time differentiations is avoided by multiplying both sides by $s^{-2}$ :

$$
m\left[2 s^{-2} X+4 s^{-1} \frac{d X}{d s}+\frac{d^{2} X}{d s^{2}}\right]=s^{-2} \frac{d^{2} U}{d s^{2}}
$$

It reads in the time domain ${ }^{12}$ :

$m\left[2 \int_{0}^{t} \int_{0}^{\sigma_{1}} x\left(\sigma_{2}\right) d \sigma_{2} d \sigma_{1}-4 \int_{0}^{t} \sigma_{1} x\left(\sigma_{1}\right) d \sigma_{1}+t^{2} x(t)\right]=\int_{0}^{t} \int_{0}^{\sigma_{1}} u\left(\sigma_{2}\right) d \sigma_{2} d \sigma_{1}$

This expression has the advantage of being completely independent of the initial conditions and it only requires the measurement of the input force $u(t)$ and of the displacement output $x(t)$, in order to compute $m$. Set $1 / m=\frac{n(t)}{d(t)}$, where

\footnotetext{
${ }^{12}$ Remember (cf. Section 3.2) that the algebraic derivative $\frac{d}{d s}$ corresponds in the time domain to the multiplication by $-t$.
} 


$$
\begin{aligned}
& n(t)=t^{2} x(t)-4 \int_{0}^{t} \sigma_{1} x\left(\sigma_{1}\right) d \sigma_{1}+2 \int_{0}^{t} \int_{0}^{\sigma_{1}} x\left(\sigma_{2}\right) d \sigma_{2} d \sigma_{1} \\
& d(t)=\int_{0}^{t} \int_{0}^{\sigma_{1}} \sigma_{2}^{2} u\left(\sigma_{2}\right) d \sigma_{2} d \sigma_{1}
\end{aligned}
$$

At time $t=0$, both the numerator and the denominator are 0 : the quotient is undetermined. We must, therefore, begin to evaluate the formula not at time 0 but at a later time, say $\epsilon \supsetneqq 0, \epsilon$ being small. Set for the estimate $1 / m_{e}$ of $1 / m$ :

$$
\frac{1}{m_{e}}=\left\{\begin{array}{lc}
\text { arbitrary } \text { for } t \in[0, \epsilon) \\
\frac{n(t)}{d(t)} & \text { for } t>\epsilon
\end{array}\right.
$$

The evaluation of the quotient is, of course, valid as long as the denominator does not go through zero.

In order to easily implement the calculations on a digital computer, and given that time integrations are needed to synthesize the numerator and the denominator expressions, we would like to give to these two quantities the character of outputs of certain dynamic systems involving differential equations. We propose then the following linear time-varying "filters":

$$
\left\{\begin{array} { l } 
{ n ( t ) = t ^ { 2 } x ( t ) + z _ { 1 } } \\
{ \dot { z } _ { 1 } = - 4 t x ( t ) + z _ { 2 } } \\
{ \dot { z } _ { 2 } = 2 x ( t ) }
\end{array} \quad \left\{\begin{array}{l}
d(t)=\eta_{1} \\
\dot{\eta}_{1}=\eta_{2} \\
\dot{\eta}_{2}=t^{2} u(t)
\end{array}\right.\right.
$$

with $z_{1}(0)=z_{2}(0)=0$ and $\eta_{1}(0)=\eta_{2}(0)=0$.

Figure 1 depicts the involved signals, i.e., the numerator $n(t)$, the denominator $d(t)$, the input $u(t)$, which is here a constant force, the output $y(t)=x(t)$, and the estimate $1 / m_{e}$ of $1 / m$. The wrong, or arbitrary, guess for the parameter value, during the time interval $[0, \epsilon)$, was taken to be $1 / m_{e}=0.5$, as it can be seen from the figure. We have set, in this case, $\epsilon=0.01 \mathrm{~s}$, but yet a smaller real value could have certainly been used. Also, we have let: $u(t)=1$ for all $t$. For the simulations, the actual value of the mass was set to $m=1 \mathrm{Kg}$.

Several distinctive features emerge from the simulations of this rather simple example:

1. The estimation of the mass parameter can be reliably achieved in a quite short amount of time that only depends on the arithmetic processor precision in being able to carry out the quotient of two very small quantities, the numerator and denominator signals.

2. The test input signal $u(t)$ being used does not necessarily exhibit the classical "persistency of excitation" requirement.

3. The estimator of the inverse mass parameter is comprised of unstable signals in both the numerator and the denominator. 

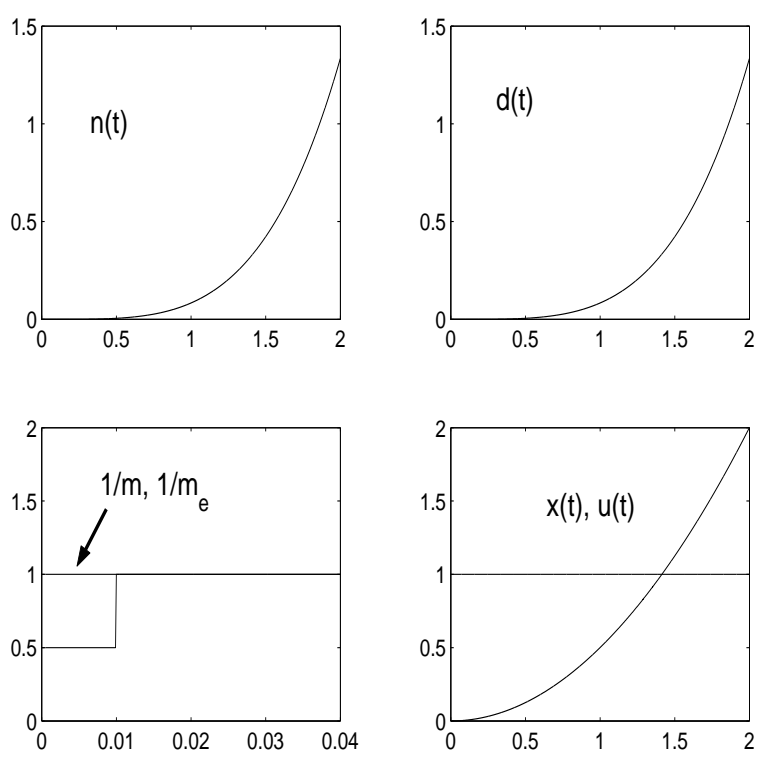

Fig. 1. Identification of the inverse mass parameter

Regarding the first observation above, we should remark that the accurate precision with which we have obtained the mass parameter is not at all surprising, due to the fact that the used formula is as exact as the model and, very importantly, because we have not included any measurement noise in our simulations. This last feature may compromise not only the precision of the computation but, also, the fast character of the identification. The second feature of not needing a persistently exciting signal is certainly an unchallenged advantage. The last negative feature regarding our internally unstable scheme may be overcome in a simple manner by prescribing the need to, at least temporarily, "switch off" the estimator right after the precise parameter estimation is obtained. The noise related aspects is quite essential. We propose below a possible approach.

\subsection{Denoising}

The expression

$$
\frac{1}{m}=\frac{n(t)}{d(t)}
$$

becomes

$$
\frac{1}{m}=\frac{\mathcal{G} \star n(t)}{\mathcal{G} \star d(t)}
$$

where 
- $\mathcal{G}$ is a low-pass filter with rational transfer function $G(s)$,

- $\star$ denotes the convolution product.

According to Sections 4.2 and 4.4 such an invariant filtering permits to attenuate zero mean highly fluctuating noises, such as the plant noise $\zeta(t)$ and the measurement noise $\xi(t)$ occurring in

$$
m \ddot{x}(t)=u(t)+\zeta(t), \quad y(t)=x(t)+\xi(t)
$$

Corresponding to the inverse mass parameter estimation we propose then the following time varying-filters, with second order integration low pass filtered outputs:

$$
\left\{\begin{array} { l } 
{ n ( t ) = z _ { 1 } } \\
{ \dot { z } _ { 1 } = z _ { 2 } } \\
{ \dot { z } _ { 2 } = ( t ) ^ { 2 } y ( t ) + z _ { 3 } } \\
{ \dot { z } _ { 3 } = - 4 t y ( t ) + z _ { 4 } } \\
{ \dot { z } _ { 4 } = 2 y ( t ) }
\end{array} \quad \left\{\begin{array}{l}
d(t)=\eta_{1} \\
\dot{\eta}_{1}=\eta_{2} \\
\dot{\eta}_{2}=\eta_{3} \\
\dot{\eta}_{3}=\eta_{4} \\
\dot{\eta}_{4}=t^{2} u(t)
\end{array}\right.\right.
$$

with $z_{1}(0)=z_{2}(0)=z_{3}(0)=z_{4}(0)=0$ and $\eta_{1}(0)=\eta_{2}(0)=\cdots=\eta_{4}(0)=0$.

We set $\xi(t)=0.02(\operatorname{rect}(t)-0.5)$ and $\zeta(t)=(\operatorname{rect}(t)-0.5)$ where $\operatorname{rect}(t)$ is a computer generated random process consisting of piecewise constant random variables uniformly distributed in the interval $[0,1]$ of the real line.
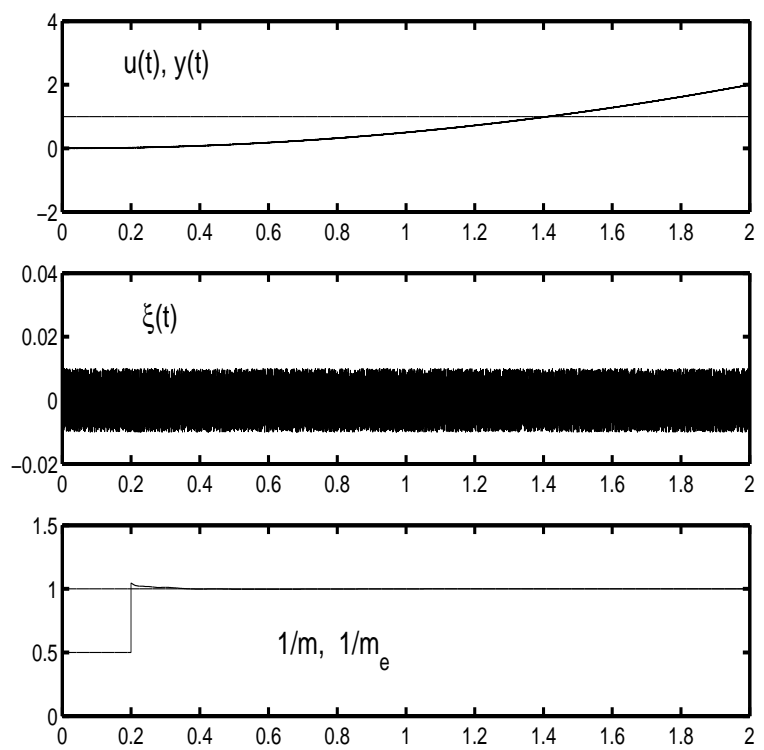

Fig. 2. Identification of the inverse mass parameter under noise measurements and using invariant filtering 
Figure 2 depicts the outcome of the invariant filtering modification of our previously proposed parameter estimation scheme for the unknown dragged mass. We note that a larger $\epsilon$ parameter was used in this instance $(\epsilon=0.2)$ to allow for a reliable quotient yielding the inverse mass, once the signal-to-noise ratio becomes important in the numerator.

\subsection{A comparison with an adaptive observer approach}

For purposes of comparison, and given that the parameter estimation problem has been cast, so far, into an open loop problem, we choose now an observer approach for the estimation of the unknown parameter (see, e.g., [24]).

Consider the state-variable representation of the mass dragging problem with a constant but unknown mass

$$
\begin{aligned}
\dot{x}_{1} & =x_{2} \\
\dot{x}_{2} & =x_{3} u \\
\dot{x}_{3} & =0 \\
y & =x_{1}
\end{aligned}
$$

where the state $x_{3}$ represents the inverse value of the unknown mass $m$. Here $u$ will be assumed to be, as before, a constant $C$. An adaptive observer is represented by the following certainty equivalence observer

$$
\begin{aligned}
& \dot{x}_{1 e}=x_{2 e}+\lambda_{3}\left(y-x_{1 e}\right) \\
& \dot{x}_{2 e}=x_{3 e} u+\lambda_{2}\left(y-x_{1 e}\right) \\
& \dot{x}_{3 e}=\lambda_{1}\left(y-x_{1 e}\right)
\end{aligned}
$$

The estimation error dynamics is given by

$$
\begin{aligned}
& \dot{e}_{1}=e_{2}-\lambda_{3} e_{1} \\
& \dot{e}_{2}=e_{3} u-\lambda_{2} e_{1} \\
& \dot{e}_{3}=-\lambda_{1} e_{1}
\end{aligned}
$$

with $e_{j}=x_{j}-x_{j e}, j=1,2,3$. Thus,

$$
\ddot{e}_{1}+\lambda_{3} \dot{e}_{1}+\lambda_{2} e_{1}+\lambda_{1} u \int_{0}^{t} e_{1}(\sigma) d \sigma=0
$$

Clearly, for $u=C$, the characteristic polynomial of the estimation error dynamics is given by

$$
p(s)=s^{3}+\lambda_{3} s^{2}+\lambda_{2} s+\lambda_{1} C=0
$$

Evidently the adaptive observer approach is limited, in this case, to those dragging maneuvers for which the constant value of $C$ is strictly positive. 
Notice that if $C$ is to be negative, as in a "pushing" task, changing the sign of $\lambda_{1}$ to a negative value (so that the term $\lambda_{1} C$ in the characteristic polynomial becomes strictly positive) simply de-stabilizes $e_{1}$. This fact severely limits the applications in the context of trajectory tracking problems, where $u$ is not constant, and also in those situations in which the steady state value of the control input is to become strictly negative, regardless of how small. Another limitation, as depicted in the simulation below, is the relatively slow convergence to the actual value of the inverse mass on the part of the estimate of $x_{3}$.
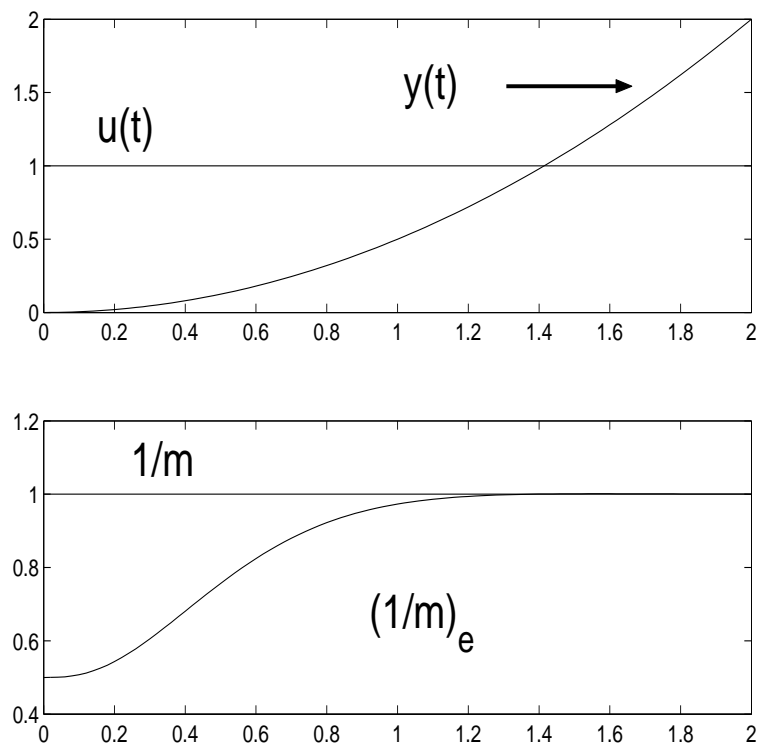

Fig. 3. Identification of the inverse mass parameter using an adaptive observer

\section{Second example: A perturbed first order system}

\subsection{Presentation}

We now turn our attention to a case where more than one unknown parameter is present in the system and, also, where the need arises for a closed loop identification. As before, regarding the control part, we resort to the certainty equivalent control method. As for how to handle several parameters, we will have to generate as many algebraic equations as unknown parameters there may be.

Consider the linear parameter-uncertain, perturbed, first order system: 


$$
\dot{y}(t)=a y(t)+b u(t)+\kappa+\xi(t)
$$

where

- $\quad a, b$ are uncertain parameters,

- $\kappa$ is an unknown constant bias,

- $\xi(t)$ is a zero mean highly fluctuating noise ${ }^{13}$.

We would like to specify a feedback control law such that the following problem finds a solution:

Devise a feedback control law that forces the output signal $y$ to follow a given reference trajectory $y^{*}(t)$, in spite of the lack of knowledge about the plant parameters $a, b$, the uncertainty about the constant perturbation $\kappa$ and the presence of the zero mean, rapidly varying, plant perturbation noise.

\subsection{A certainty equivalence controller}

If the parameters $a$ and $b$ were perfectly known, and if there existed no plant perturbation noise, i.e., if $\xi(t) \equiv 0$, the following classical proportional integral controller may be used given its known robustness with respect to a constant, but unknown, perturbation:

$$
u=\frac{1}{b}\left[\dot{y}^{*}(t)-a y-c_{1}\left(y-y^{*}(t)\right)-c_{0} \int_{0}^{t}\left(y(\sigma)-y^{*}(\sigma)\right) d \sigma\right]
$$

Set $e=y-y^{*}(t)$. The characteristic polynomial of the closed loop tracking error dynamics is given by $p(s)=s^{2}+c_{1} s+c_{0}$. With a suitable choice of the design parameters $c_{0}, c_{1}$, the roots of $p(s)$ are all strictly located in the left portion of the complex plane. The tracking task is asymptotically accomplished.

We must therefore concentrate our efforts on obtaining the right values of $a$ and $b$.

\subsection{Parameter identification}

Assume again that $\xi(t) \equiv 0$. We try to generate a linear system of equations for the unknown parameters $a$ and $b$. This system should be independent of the plant initial condition, and also, of course, independent of the constant perturbation, $\kappa$ and, moreover, it should rely only on knowledge of the input $u$ and the output $y$ signals.

Operational calculus yields:

$$
s Y-y_{0}=a Y+b U+\frac{\kappa}{s}
$$

$\overline{13}$ One might replace $\kappa$ and $\xi(t)$ by a highly fluctuating noise of constant but unknown mean (cf. [6]). 
Multiply both sides by $s$ :

$$
s^{2} Y-s y_{0}=a s Y+b s U+\kappa
$$

Differentiating twice with respect to $s$ gets rid of the presence of the initial condition and, also, of the influence of the unknown parameter $\kappa$. We obtain, after some algebraic manipulations:

$$
a\left[2 \frac{d Y}{d s}+s \frac{d^{2} Y}{d s^{2}}\right]+b\left[2 \frac{d U}{d s}+s \frac{d^{2} U}{d s^{2}}\right]=2 Y+4 s \frac{d Y}{d s}+s^{2} \frac{d^{2} Y}{d s^{2}}
$$

Multiplying by $s^{-2}$ to avoid time differentiations in the time domain, we get

$$
\begin{aligned}
& a\left[2 s^{-2} \frac{d Y}{d s}+s^{-1} \frac{d^{2} Y}{d s^{2}}\right]+b\left[2 s^{-2} \frac{d U}{d s}+s^{-1} \frac{d^{2} U}{d s^{2}}\right] \\
& =2 s^{-2} Y+4 s^{-1} \frac{d Y}{d s}+\frac{d^{2} Y}{d s^{2}}
\end{aligned}
$$

It reads in the time-domain:

$$
\begin{aligned}
& {\left[\int_{0}^{t} \sigma_{1}^{2} y\left(\sigma_{1}\right) d \sigma_{1}-2 \int_{0}^{t} \int_{0}^{\sigma_{1}} \sigma_{2} y\left(\sigma_{2}\right) d \sigma_{2} d \sigma_{1}\right] a} \\
& +\left[\int_{0}^{t} \sigma_{1}^{2} u\left(\sigma_{1}\right) d \sigma_{1}-2 \int_{0}^{t} \int_{0}^{\sigma_{1}} \sigma_{2} u\left(\sigma_{2}\right) d \sigma_{2} d \sigma_{1}\right] b \\
& =t^{2} y(t)-4 \int_{0}^{t} \sigma_{1} y\left(\sigma_{1}\right) d \sigma_{1}+2 \int_{0}^{t} \int_{0}^{\sigma_{1}} y\left(\sigma_{2}\right) d \sigma_{2} d \sigma_{1}
\end{aligned}
$$

Integrating once more, we obtain a linear system for the constant parameters $a$ and $b$. We arrive at a linear time-varying equation:

$$
P(t)\left[\begin{array}{l}
a \\
b
\end{array}\right]=q(t)
$$

The $2 \times 2$ matrix $P(t)$ reads:

$$
P(t)=\left[\begin{array}{ll}
p_{11}(t) & p_{12}(t) \\
p_{21}(t) & p_{22}(t)
\end{array}\right]
$$

where

$$
\begin{aligned}
& p_{11}(t)=\int_{0}^{t} \sigma^{2} y d \sigma-2 \int_{0}^{t} \int_{0}^{\sigma} \lambda y d \lambda d \sigma \\
& p_{12}(t)=\int_{0}^{t} \sigma^{2} u d \sigma-2 \int_{0}^{t} \int_{0}^{\sigma} \lambda u d \lambda d \sigma \\
& p_{21}(t)=\int_{0}^{t} \int_{0}^{\sigma} \lambda^{2} y d \lambda \sigma-2 \int_{0}^{t} \int_{0}^{\sigma} \int_{0}^{\lambda} \rho y d \rho d \lambda d \sigma \\
& p_{22}(t)=\int_{0}^{t} \int_{0}^{\sigma} \lambda^{2} u d \lambda \sigma-2 \int_{0}^{t} \int_{0}^{\sigma} \int_{0}^{\lambda} \rho u d \rho d \lambda d \sigma
\end{aligned}
$$


The column vector $q(t)$ is given by

$$
q(t)=\left[\begin{array}{c}
t^{2} y-4 \int_{0}^{t} \sigma y d \sigma+2 \int_{0}^{t} \int_{0}^{\sigma} y d \lambda d \sigma \\
\int_{0}^{t} \sigma^{2} y d \sigma-4 \int_{0}^{t} \int_{0}^{\sigma} \lambda y d \lambda d \sigma+2 \int_{0}^{t} \int_{0}^{\sigma} \int_{0}^{\lambda} y d \lambda d \sigma d \rho
\end{array}\right]
$$

The matrix $P(t)$ and the vector $q(t)$ are equal to 0 at time $t=0$. Nevertheless, it is easy to verify that the matrix $P(t)$ is, indeed, invertible at a small time, $t=\epsilon>0$.

Under the noise-free circumstances, we may, then compute $a$ and $b$ exactly, at time $t=\epsilon>0$, regardless of the constant perturbation input $\kappa$, and, moreover, for any initial condition on the plant output $y$.

A certainty equivalence controller, of the form (20), is proposed as follows:

$$
u=\frac{1}{b_{e}}\left[\dot{y}^{*}(t)-a_{e} y-k_{1}\left(y-y^{*}(t)\right)-k_{0} \int_{0}^{t}\left(y(\sigma)-y^{*}(\sigma)\right) d \sigma\right]
$$

with

$$
\left[\begin{array}{l}
a_{e} \\
b_{e}
\end{array}\right]=\left\{\begin{array}{l}
\text { arbitrary, with } b_{e} \neq 0 \text { for } t \in[0, \epsilon) \\
P^{-1}(t) q(t) \quad \text { for } t \in[\epsilon,+\infty)
\end{array}\right.
$$

\subsection{Noise-free simulation results}

Figure 4 depicts the fast adaptation system response in a rest to rest trajectory tracking task. As it can be seen, the determination of the system parameters happens quite fast, in approximately $4 \times 10^{-3} \mathrm{~s}$. The absence of measurement and plant noises certainly makes the algebraic estimation task quite precise and rather fast. The integral action on the proposed certainty equivalence controller annihilates the effects of the unknown constant perturbation input while our estimation technique is shown to be totally independent of the constant perturbation input amplitude.

\subsection{Noisy measurements and plant perturbations}

To carry out our previously proposed algebraic parameter estimation approach to fast adaptive control, we considered the following intimately related perturbed system:

$$
\dot{x}=a x+b u+k+\eta(t), \quad y(t)=x(t)+\xi(t)
$$

where $\eta(t)$ and $\xi(t)$ are zero mean computer generated noises consisting of a sequence of piecewise constant random variables uniformly distributed in the interval $[-0.5 R, 0.5 R]$.

For the case of measurement noises, the same computational algorithm was used but now including an invariant filtering strategy. We low-pass filtered both members of each one of the algebraic equations derived before for the 

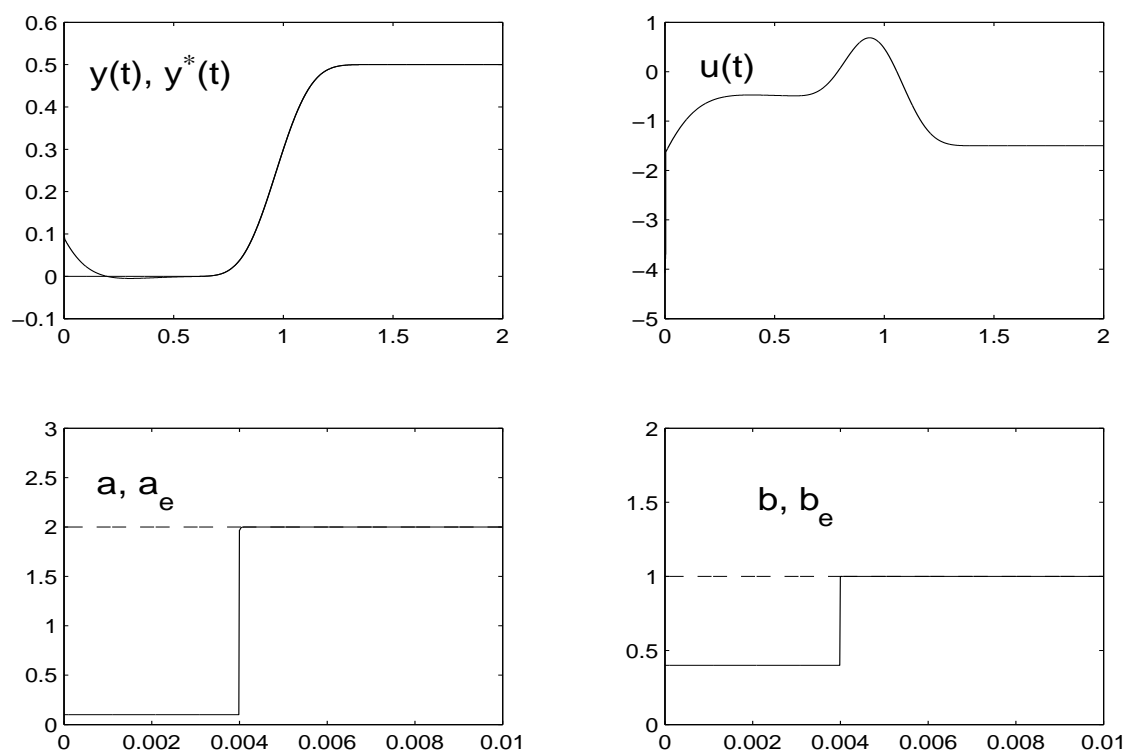

Fig. 4. System response, parameter determination, control input

on line calculation of the parameters. A second order integration was used in each case. In the simulation shown in the next figure, we have assumed a zero mean computer generated measurement noise of significant amplitude. The output signal, and the control input signal do exhibit the influence of the measurement noise but the parameter estimates converge quite precisely and fast enough to the actual value of the parameters. The computation time is substantially increased in the noisy case. Nevertheless, the estimation of the unknown parameters is still quite accurate.

\subsection{Simulation results with noises}

Figure 5 depicts the performance of the algebraic parameter identifier including invariant filtering along with the systems response in a rest to rest trajectory tracking task and the evolution of the applied feedback control input. For the measurement noise $\xi(t)$ we have chosen $R$ to be 0.01 and for the plant system noise $\eta$, the corresponding $R$ value was set to be 0.1

\subsection{Comparison with adaptive control}

Adaptive control is usually approached from the viewpoint of Lyapunov stability theory via the synthesis of a suitable parameter update law derived on the basis of the behaviour around a closed loop trajectory of the time derivative of a Lyapunov function which includes a quadratic parameter estimation 

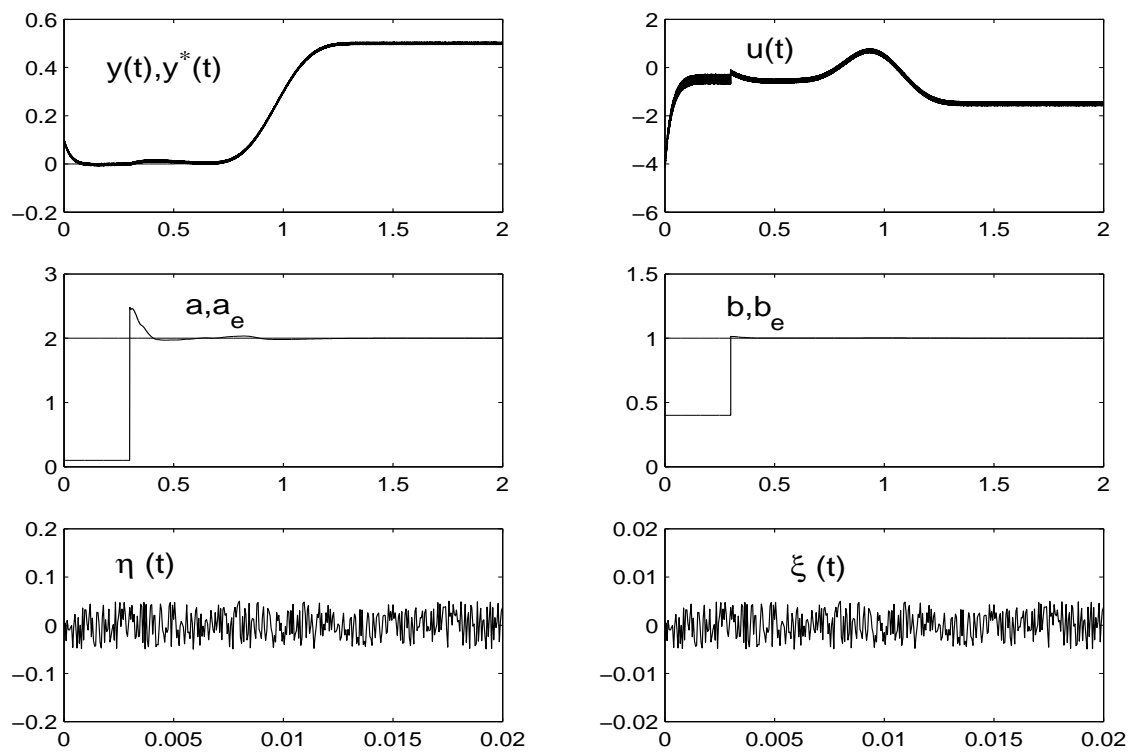

Fig. 5. System response, parameter determination and control input

error term. The feedback law is proposed as a certainty equivalent controller. The adaptation mechanism is derived by enforcing asymptotic stability of the closed loop controlled system. The literature on the topic of adaptive control using Lyapunov arguments is certainly overwhelming. For further details, we refer the reader to popular references, like [1, 14, 23, 27].

We deal with the same system as in the previous section

$$
\dot{y}=a y+b u+\kappa
$$

A nominal desired trajectory for the system $y^{*}(t)$ demands the existence of a nominal control input $u^{*}(t)$ which satisfies the dynamics of the unperturbed system.

$$
\dot{y}^{*}(t)=a y^{*}(t)+b u^{*}(t)
$$

The tracking error dynamics is then given by

$$
\dot{e}=a e+b e_{u}+\kappa
$$

where $e=y-y^{*}(t)$ and $e_{u}=u^{*}(t)$. A certainty equivalence control, using estimated values of the unknown parameters, is given by

$$
e_{u}=u-u^{*}(t)=\frac{1}{b_{e}}\left[-a_{e} e-k_{1} e-k_{0} \int_{0}^{t} e(\sigma) d \sigma\right]
$$

where $a_{e}$ and $b_{e}$ are the estimated values of $a$ and $b$. 
The closed loop system, after some algebraic manipulations, results in

$$
\begin{aligned}
\dot{e}+k_{1} e+k_{0} \rho & =\left(a-a_{e}\right) e-\frac{1}{b_{e}}\left(b-b_{e}\right)\left[\left(k_{1}-a_{e}\right) e+k_{0} \int_{0}^{t} e(\sigma) d \sigma\right] \\
\dot{\rho} & =e(\sigma), \quad \rho(0)=-\frac{\kappa}{k_{0}}
\end{aligned}
$$

Taking as a Lyapunov function candidate the following positive definite function

$$
V\left(e, \rho, a-a_{e}, b-b_{e}\right)=\frac{1}{2} e^{2}+\frac{k_{0}}{2} \rho^{2}+\frac{1}{2 \gamma}\left(a-a_{e}\right)^{2}+\frac{1}{\beta}\left(b-b_{e}\right)^{2}
$$

we find that the choice of the estimated values of $a$ and $b$ according to the following parameter update law

$$
\begin{aligned}
\dot{a}_{e} & =\gamma e^{2} \\
\frac{d}{d t}\left[b_{e}\right]^{2} & =\beta\left[\left(k_{1}-a_{e}\right) e^{2}+k_{0} e \int_{0}^{t} e(\sigma) d \sigma\right]
\end{aligned}
$$

leads to the following expression for the time derivative of $V(e)$ along the trajectories of the controlled system:

$$
\dot{V}(e)=-k_{1} e^{2} \leq 0
$$

The non positivity of $\dot{V}(e)$ implies that $V(e)$ is bounded. It is also clear that $\dot{V}(e)$ is absolutely continuous. It follows, according to Barabarat's lemma, that $\dot{V}(e)$ asymptotically converges to zero. Hence $e$ tends to zero. It is also clear that the convergence of $a_{e}$ and $b_{e}$ to their actual values cannot be guaranteed. As a consequence of this, the value of $k_{1}$ must be chosen sufficiently large so that $a_{e}$ does not cause an instability in the dynamics of $b_{e}^{2}$. But this in turn depends on the transient of the tracking error $e$. The approach may suffer severe limitations in trajectory tracking tasks.

\subsection{Simulations for the adaptive scheme}

Figure 6 depicts the performance of the designed adaptive feedback control law in the same trajectory tracking task of the previous algebraic approach example. Although the scheme manages to accomplish the trajectory tracking task with rather low quality, the scheme fails to produce an accurate estimate of the unknown parameters. The values of the parameter update gains were chosen to be $\gamma=100$ and $\beta=1.25$. The values of $a$ and $b$ used in the simulations were the same as before $a=2$ and $b=1$. If the rest to rest maneuver entitles a higher final equilibrium value, say of 1 , the scheme completely fails. 

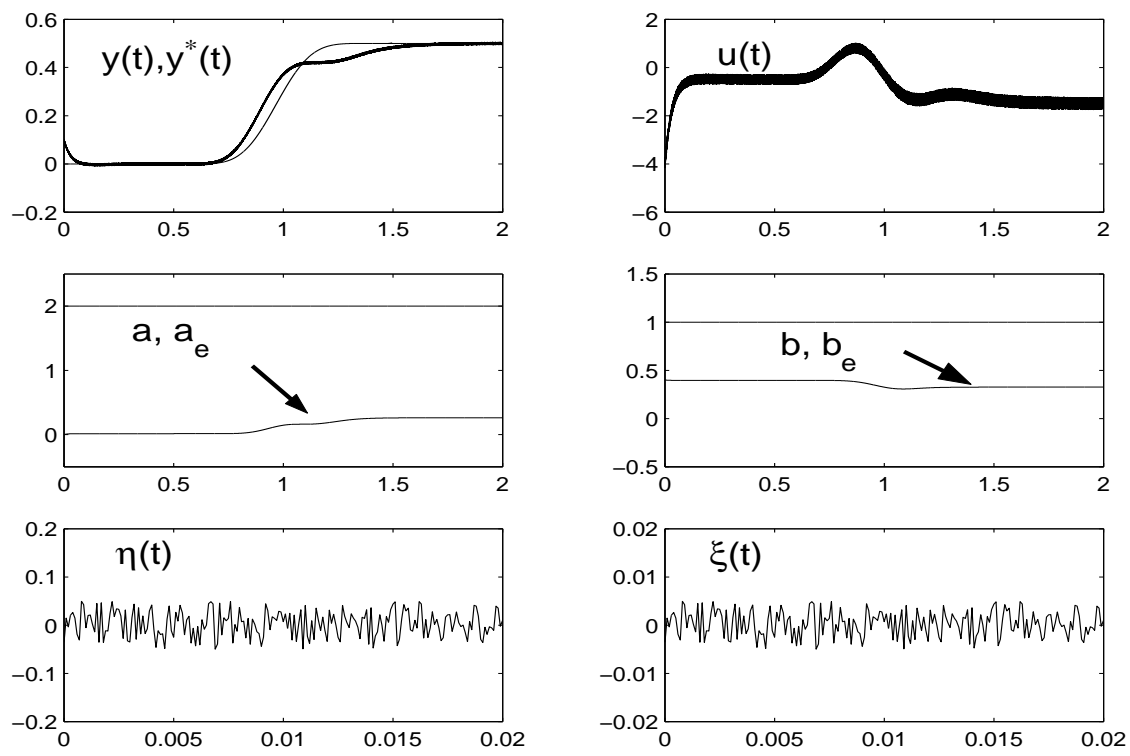

Fig. 6. Performance of adaptive control approach in a trajectory tracking task for the uncertain system

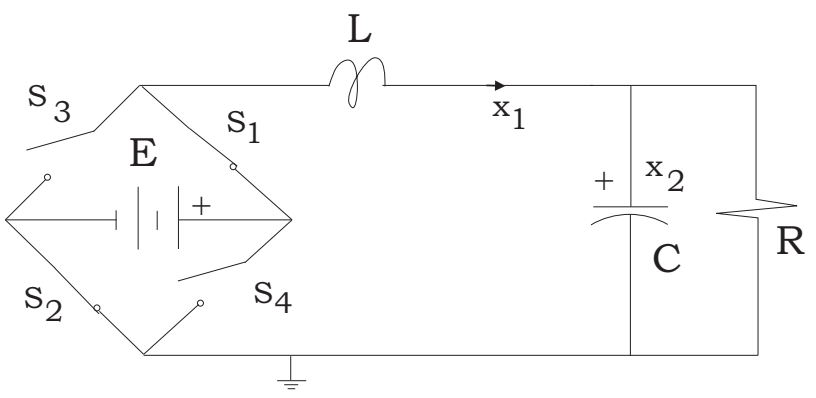

Fig. 7. The double bridge Buck converter

\section{Third example: A double bridge buck converter}

The several electronic switches in Figure 7 take position values according to

$$
\left\{\begin{array}{l}
u=1, S_{1}=O N, S_{2}=O N, S_{3}=O F F, S_{4}=O F F \\
u=0, S_{1}=O F F, S_{2}=O F F, S_{3}=O N, S_{4}=O N
\end{array}\right.
$$

Consider the following (average) model of a double bridge buck-converter ${ }^{14}$ :

${ }^{14}$ See [30] for further details on this and other power converters. For a closely related on-line adaptive identification case on the same converter, see [29]. 


$$
\begin{aligned}
L \dot{x}_{1} & =-x_{2}+\mu E \\
C \dot{x}_{2} & =x_{1}-\frac{x_{2}}{R} \\
y & =x_{2}
\end{aligned}
$$

where

- $x_{1}$ is the inductor current,

- $x_{2}$ represents the capacitor voltage.

The average control input $\mu$ is assumed to take values in the closed interval $[-1,1]$. This variable actually represents, in absolute value, the duty ratio of the switch positions. The parameters $L, C, R$ and $E$ are assumed to be unknown.

\subsection{An input-output model}

Eliminating the state variable $x_{1}$ yields

$$
\ddot{y}+\gamma_{1} \dot{y}+\gamma_{0} y=\gamma \mu
$$

where the parameters $\gamma_{1}=\frac{1}{R C}, \gamma_{0}=\frac{1}{L C}, \gamma=\frac{E}{L C}$ are linearly identifiable according to Section 3.4. Estimating those parameters permits to control the system without knowing the values of $L, C, R$ and $E$.

Remark 5. It is straightforward to check that $L, C, R, E$ are not simultaneously identifiable.

\subsection{Problem Formulation}

It is required to design an output feedback controller, possibly of dynamic nature, which induces in the uncertain system, representing the double bridge buck converter average model, an exponentially asymptotic convergence of the output signal $y$ towards the desired reference signal $y^{*}(t)$. In other words, we want:

$$
y \rightarrow y^{*}(t) \text { exponentially }
$$

\subsection{A certainty equivalence controller}

We proceed to design the controller as if these parameters were all perfectly known. We propose the following certainty equivalence generalized GPI controller $^{15}$

$$
\mu=\mu^{*}(t)-\mathcal{G} \star\left(y-y^{*}(t)\right)
$$

where

${ }^{15}$ GPI controllers were introduced in [10] for linear systems in terms of integral reconstructors yielding states in terms of iterated integrals of inputs and outputs. It can be shown, with some work, that such controllers are also equivalent to classical compensation networks of which Equation (21) is just an example. 
- $\star$ denotes the convolution product,

- the transfer function of $\mathcal{G}$ is

$$
\frac{1}{\gamma}\left\{\frac{\left[\gamma_{1}\left(\gamma_{1}-c_{1}\right)+c_{0}-\gamma_{0}\right] s+\gamma_{0}\left(\gamma_{1}-c_{1}\right)+c_{-1}}{s+\left(c_{1}-\gamma_{1}\right)}\right\}
$$

\subsection{Closed loop behavior}

The closed loop behavior of the tracking error, were the parameters perfectly known, is given by the following linear dynamics

$$
\ddot{e}_{y}+c_{1} \dot{e}_{y}+c_{0} e_{y}+c_{-1} \int_{0}^{t} e_{y}(\sigma) d \sigma=0
$$

where $e_{y}(t)=y(t)-y^{*}(t)$ is the trajectory tracking error.

The appropriate choice of the coefficients $\left\{c_{1}, c_{0}, c_{-1}\right\}$, in the characteristic polynomial of the tracking error dynamics, turns it into a Hurwitz polynomial with the associated asymptotically exponentially stable nature of the origin of coordinates of the natural tracking error state space $\left\{e_{y}=0, \dot{e}_{y}=0, \ddot{e}_{y}=0\right\}$.

Under the assumption of perfect parameter knowledge we obtain, modulo control input saturations,

$$
e_{y}(t) \rightarrow 0 \quad \text { exponentially }
$$

The problem becomes now one of accurate determination of the unknown parameters of the system as required by the proposed GPI controller

\subsection{Algebraic determination of the unknown parameters}

Consider the average input output model of the converter system

$$
\ddot{y}+\gamma_{1} \dot{y}+\gamma_{0} y=\gamma \mu
$$

In the notation of operational calculus, we have:

$$
s^{2} Y-s y_{0}-\dot{y}_{0}+\gamma_{1}\left(s Y-y_{0}\right)+\gamma_{0} Y=\gamma U
$$

Taking derivatives with respect to $s$, twice, we obtain:

$$
\left(s^{2}+\gamma_{1} s+\gamma_{0}\right) \frac{d^{2} Y}{d s^{2}}+\left(4 s+2 \gamma_{1}\right) \frac{d Y}{d s}+2 Y=\gamma \frac{d^{2} U}{d s^{2}}
$$

This last expression may be rewritten as follows:

$$
\begin{gathered}
{\left[s \frac{d^{2} Y}{d s^{2}}+2 \frac{d Y}{d s}\right] \gamma_{1}+\left[\frac{d^{2} Y}{d s^{2}}\right] \gamma_{0}-\left[\frac{d^{2} U}{d s^{2}}\right] \gamma=} \\
-s^{2} \frac{d^{2} Y}{d s^{2}}-4 s \frac{d Y}{d s}-2 Y
\end{gathered}
$$


Multiplying out by a sufficient power of $s^{-1}$, say by $s^{-4}$, so that an invariant filtering effect is obtained, we also get rid of possible derivations in the time domain. We obtain:

$$
\begin{gathered}
{\left[s^{-3} \frac{d^{2} Y}{d s^{2}}+2 s^{-4} \frac{d Y}{d s}\right] \gamma_{1}+\left[s^{-4} \frac{d^{2} Y}{d s^{2}}\right] \gamma_{0}-\left[s^{-4} \frac{d^{2} U}{d s^{2}}\right] \gamma=} \\
-\left[s^{-2} \frac{d^{2} Y}{d s^{2}}+4 s^{-3} \frac{d Y}{d s}+2 s^{-4} Y\right]
\end{gathered}
$$

Reverting the previous expression to the time domain, we obtain a linear equation, with time-varying coefficients, in three unknowns $\left\{\gamma_{1}, \gamma_{0}, \gamma\right\}$. We write such an equation as

$$
p_{11}(t) \gamma_{1}+p_{12}(t) \gamma_{0}+p_{13}(t) \gamma=q_{1}(t)
$$

We conform a system of three equations in three unknowns by simply adjoining to the previous equation its first integral and its iterated integral, i.e.,

$$
\begin{aligned}
p_{11}(t) \gamma_{1}+p_{12}(t) \gamma_{0}+p_{13}(t) \gamma & =q_{1}(t) \\
\left(\int p_{11}(t)\right) \gamma_{1}+\left(\int p_{12}(t)\right) \gamma_{0}+\left(\int p_{13}(t)\right) \gamma & =\left(\int q_{1}(t)\right) \\
\left(\int^{(2)} p_{11}(t)\right) \gamma_{1}+\left(\int^{(2)} p_{12}(t)\right) \gamma_{0}+\left(\int^{(2)} p_{13}(t)\right) \gamma & =\left(\int^{(2)} q_{1}(t)\right)
\end{aligned}
$$

This linear system of equation allows us to determine $\gamma_{1}, \gamma_{0}$ and $\gamma$ for $t \geq \epsilon$ with $\epsilon$ being a very small positive real number.

\subsection{Simulation Results}

We considered the average model of a double bridge buck converter with the following (unknown) parameters

$$
R=39.52 \Omega, \quad L=1 \mathrm{mH}, \quad C=1 \mu \mathrm{F}, \quad E=30 \text { Volts }
$$

It is desired that the average output voltage signal tracks a rest to rest trajectory starting at $21.0 \mathrm{~V}$ and landing at 9.0 Volts in approximately 0.474 ms. The tracking maneuver is to start at $t_{\text {init }}=0.158 \mathrm{~ms}$. and it ends at $t_{f}=0.632 \mathrm{~ms}$. It was assumed that the output voltage could be measured through an additive noise process simulated with a computer generated sequence of random variables uniformly distributed in the interval $A[-0.5,0.5]$ with the factor $A$ taken to be $A=0.3$. 

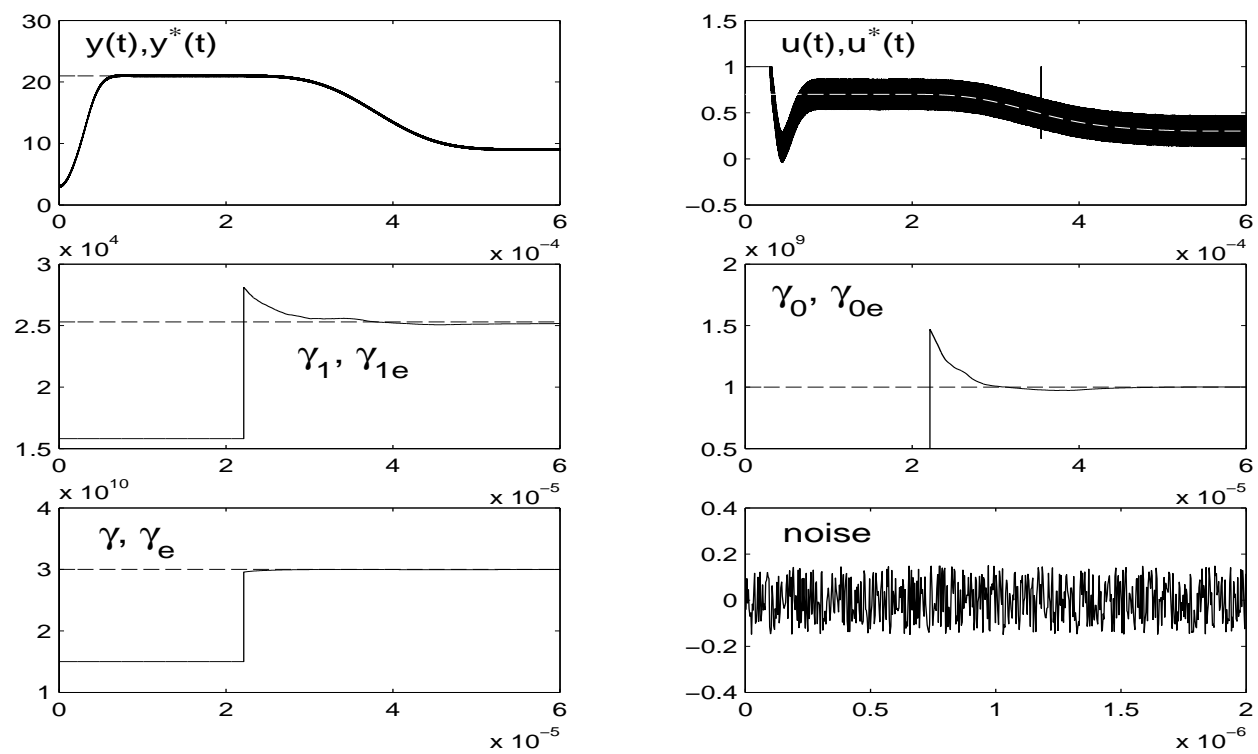

Fig. 8. Closed loop average converter response with on-line identification of all linearly identifiable system parameters

Figure 8 depicts the simulated closed loop performance of the GPI controlled double bridge boost converter along with the performance of the proposed algebraic parameter estimator. The value of $\epsilon$ used to avoid the singularity of the formulae at time $t=0$ was taken to be $22 \mu \mathrm{s}$. The three parameters are identified, rather accurately, in approximately $30 \mu$ s. Once identified, the value of the parameters is immediately substituted on the GPI feedback control law.

We tested our fast adaptive estimation algorithm using now a switched control signal for the control input whose average coincides with the previous control input. This is achieved using a double sided Sigma-Delta modulator described by the following discontinuous dynamics:

$$
\dot{z}=\mu-u, \quad u=\frac{1}{2}[\operatorname{sign}(\mu)+\operatorname{sign}(z)]
$$

The actual control input signal $u$ being used in the identification algorithm is now a high frequency signal actively switching and taking values in the discrete set $\{-1,0,+1\}$. As it can be inferred from Figure 9 the algebraic identifier works perfectly well with this input. In this instance, the maneuver entitled a trajectory tracking task, with the same time duration constraints as before, taking the output voltage from an initial equilibrium of 21 Volts towards a final equilibrium of -9 Volts.

Figure 9 shows the closed loop response for the trajectory tracking task of the switched input model as well as the precision and rapidity of the unknown 

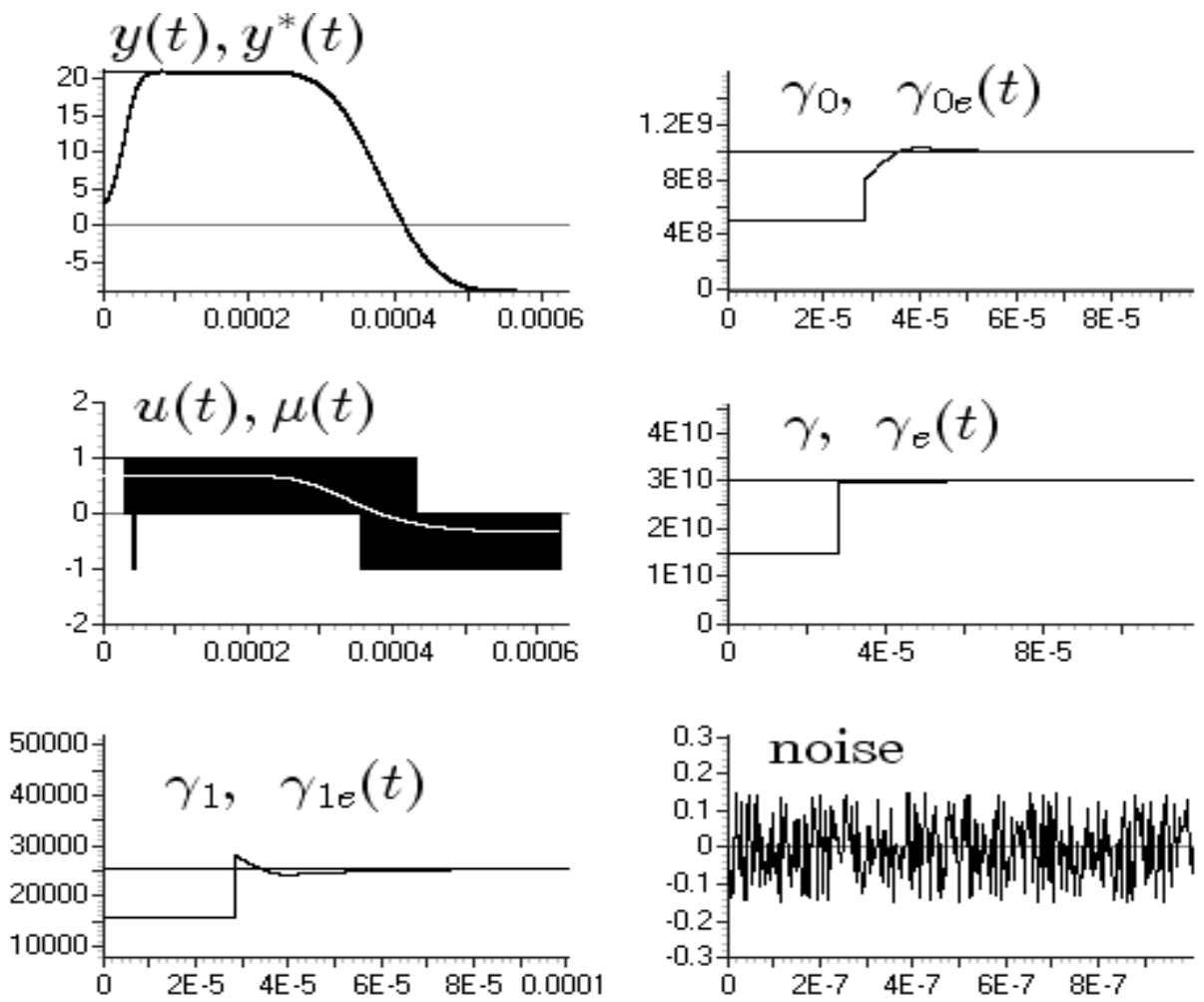

Fig. 9. Closed loop switched converter response with on-line identification of all linearly identifiable system parameters

parameter estimation process. The output voltage signal is also assumed to be measured through an additive noisy means. A sample of the noise process is also depicted in the figure. The actual bang-bang control input is shown along with the nominal value of the average control input.

\section{Conclusion}

It is a delicate matter to compare our theoretical techniques and results with today's parametric identification of linear continuous-time systems (see, e.g., $[13,15,18,19,26,33]$ and the references therein), which is perhaps less developed than its discrete-time counterpart, but nevertheless makes also generally a heavy utilization of statistical methods ${ }^{16}$. Let us stress that all those approaches seem to rest on standpoints and therefore on mathematical tools which are rather far from ours. It is our belief that the only fair way for achieving such a comparison is provided by examples. We do hope that the readers

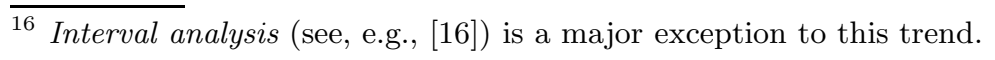


will be convinced by the numerous case-studies examined in this chapter and in [11].

The above techniques and results may be generalized to linear state reconstructors [12], to linear diagnosis [8], and to parametric identification of linear discrete-time systems [7]. See, e.g., the references in [6] for their extensions to non-linear systems as well as to signal processing.

\section{References}

1. K.J. Astrom and B. Wittenmark. Adaptive Control. Addison Wesley, New York, $2^{\text {nd }}$ edition, 1995.

2. H. Bourlès. Systèmes linéaires : de la modélisation à la commande. Hermès, Paris, 2006.

3. G. Doetsch. Introduction to the Theory and Application of the Laplace Transform. Translated from the German. Springer, Berlin, 1974.

4. M. Fliess. Some basic structural properties of generalized linear systems. Systems Control Lett., 15:391-396, 1990.

5. M. Fliess. Une interprétation algébrique de la transformation de Laplace et des matrices de transfert. Linear Algebra Appl., 203-204:429-442, 1994.

6. M. Fliess. Analyse non standard du bruit. C.R. Acad. Sci. Paris Ser. I, 342:797$802,2006$.

7. M. Fliess, S. Fuchshumer, K. Schlacher, and H. Sira-Ramírez. Discretetime linear parametric identification: An algebraic approach. In $2^{e}$ Journée Identif. Modél. Expérim. - JIME'2006, Poitiers, 2006 (available at http://hal.inria.fr/inria-00105673).

8. M. Fliess, C. Join, and H. Sira-Ramírez. Robust residual generation for linear fault diagnosis: an algebraic setting with examples. Internat. J. Control, $77: 1223-1242,2004$.

9. M. Fliess and R. Marquez. Continuous-time linear predictive control and flatness: A module-theoretic setting with examples. Internat. J. Control, 73:606$623,2000$.

10. M. Fliess, R. Marquez, E. Delaleau, and H. Sira-Ramírez. Correcteurs proportionnels-intégraux généralisés. ESAIM Control Optim. Calc. Variat., $7: 23-41,2002$.

11. M. Fliess and H. Sira-Ramírez. An algebraic framework for linear identification. ESAIM Control Optim. Calc. Variat., 9:151-168, 2003.

12. M. Fliess and H. Sira-Ramírez. Reconstructeurs d'état. C.R. Acad. Sci. Paris Ser. I, 338:91-96, 2004.

13. H. Garnier, M. Mensler, and A. Richard. Continuous-time model identification from sampled data: implementation issues and performance evaluation. Internat. J. Control, 76:1337-1357, 2003.

14. G. Goodwin and D. Mayne. A parameter estimation perspective to continuous time model reference adaptive control. Automatica, 23:57-70, 1987.

15. P. van den Hof. Closed-loop issues in system identification. Annals Reviews Control, 22:173-1186, 1998.

16. L. Jaulin, M. Kieffer, O. Didrit, and E. Walter. Applied Interval: With Examples in Parameter and State Estimation, Robust Control and Robotics. Springer, Berlin, 2001. 
17. S. Lang. Algebra. Springer, Berlin, $3^{\text {rd }}$ edition, 2002.

18. E.K. Larsson, M. Mossberg, and T. Soderstrom. An overview of important practical aspects of continuous-time arma system identification. J. Circuits Systems Signal Proc., 25:17-46, 2006.

19. K. Mahata and H. Garnier. Identification of contnuous-time errors-in-variable models. Automatica, 42:1470-1490, 2006.

20. J. McConnel and J. Robson. Noncommutative Noetherian Rings. Amer. Math. Soc., Providence, RI, 2000.

21. J. Mikusinski. Operational Calculus. vol. 1, PWN \& Pergamon, Warsaw \& Oxford, $2^{\text {nd }}$ edition, 1983.

22. J. Mikusinski and T. Boehme. Operational Calculus. vol. 2, PWN \& Pergamon, Warsaw \& Oxford, $2^{\text {nd }}$ edition, 1987.

23. K. Narendra and A. Annaswamy. Stable Adaptive Systems. Prentice Hall, Englewood Cliffs, 1989.

24. M. Phan, L. Horta, and R. Longman. Linear system identification via an asymptotically stable observer. J. of Opt. Theory and Appl., 79:59-86, 1993.

25. B. van der Pol and H. Bremmer. Operational Calculus Based on the Two-Sided Laplace Integral. Cambridge University Press, Cambridge, $2^{\text {nd }}$ edition, 1955.

26. T.I. Salsbury. Continuous-time model identification for closed loop control performance assessment. Control Engineering Pract., 15:109-121, 2007.

27. S. Sastry and M. Bodson. Adaptive Systems: Stability, Convergence and Robustness. Prentice Hall, Englewood Cliffs, 1989.

28. H. Sira-Ramírez and S.K. Agrawal. Differentially Flat Systems. Marcel Dekker, New York, 2004.

29. H. Sira-Ramírez, E. Fossas, and M. Fliess. An algebraic, on-line, parameter identification approach to uncertain dc-to-ac power conversion. In Proc. $41^{\text {st }}$ IEEE Conf. Decision Control, Las Vegas, Nevada, 2002.

30. H. Sira-Ramírez and R. Silva-Ortigoza. Control Design Techniques in Power Electronics Devices. Springer, London, 2006.

31. J.-C. Trigeassou. Identification et commande des processus mono-entrée monosortie par la méthode des moments - Expérimentation sur calculatrice programmable. Thèse $3^{e}$ cycle, Université de Nantes, 1980.

32. K. Yosida. Operational Calculus. Springer, New York, 1984.

33. P.C. Young. Parameter estimation for continuous-time models - a survey. $A u$ tomatica, 17:23-39, 1981. 